\title{
Synthesis of Carbon Encapsulated Mono- and Multi-Iron Nanoparticles
}

\author{
M. Reza Sanaee and Enric Bertran \\ FEMAN Grupo, Instituto de Nanociencia y Nanotecnología (IN2UB), Departament de Física Aplicada i Òptica, \\ Universitat de Barcelona, Martí i Franquès 1, 08028 Barcelona, Spain
}

Correspondence should be addressed to M. Reza Sanaee; mreza_sanaee@yahoo.com

Received 16 December 2014; Revised 8 February 2015; Accepted 19 February 2015

Academic Editor: Dan Xia

Copyright (C) 2015 M. R. Sanaee and E. Bertran. This is an open access article distributed under the Creative Commons Attribution License, which permits unrestricted use, distribution, and reproduction in any medium, provided the original work is properly cited.

\begin{abstract}
Core-shell nanostructures of carbon encapsulated iron nanoparticles (CEINPs) show unique properties and technological applications, because carbon shell provides extreme chemical stability and protects pure iron core against oxidation without impairing the possibility of functionalization of the carbon surface. Enhancing iron core magnetic properties and, in parallel, improving carbon shell sealing are the two major challenges in the synthesis of CEINPs. Here, we present the synthesis of both CEINPs and a new carbon encapsulated multi-iron nanoparticle by a new modified arc discharge reactor. The nanoparticle size, composition, and crystallinity and the magnetic properties have been studied. The morphological properties were observed by scanning electron microscopy and transmission electron microscopy. In order to evaluate carbon shell protection, the iron cores were characterized by selected area diffraction and fast Fourier transform techniques as well as by electron energy loss and energy dispersive X-ray spectroscopies. Afterward, the magnetic properties were investigated using a superconducting quantum interference device. As main results, spherical, oval, and multi-iron cores were controllably synthesized by this new modified arc discharge method. The carbon shell with high crystallinity exhibited sufficient protection against oxidation of pure iron cores. The presented results also provided new elements for understanding the growth mechanism of iron core and carbon shell.
\end{abstract}

\section{Introduction}

There has been an increasing interest in fabrication of magnetic based nanomaterials due to their potential applications in data storage [1], Li-ion battery [2], highly sensitive magnetic sensors [3], and spintronics devices [4]. Moreover, magnetic nanoparticles are attracting attention in both the medical and biological fields for applications including magnetic separation of biological entities [5], tissue engineering [6], food analysis [7], therapeutic drug delivery [8], hyperthermia for tumor therapy [9], contrast enhancement agents for magnetic resonance imaging applications $[10,11]$, water purification [12], and catalysis [13, 14]. Most of the studies in these areas focus on particles based on oxides since magnetic nanoparticles which are based on pure metallic materials are very sensitive to oxidation given their high specific surface area and reactivity [15].
The key point of using magnetic nanoparticles is the possibility and capability to control them by external magnetic field. Naked metallic nanoparticles are chemically highly active and are easily oxidized in air, resulting in negative effects on magnetic properties. To avoid such consequences, well developed graphitic carbon layers can provide an effective barrier to maintain magnetic properties. Coating metallic cores by silica [16], carbon [17-20], and precious-metal [21] is an example of popular inorganic coating approaches. Compared to the polymer, silica, or Au layer, the carbon layer has many advantages, such as higher chemical and thermal stability, better conductivity, and higher biocompatibility [22-24]. Moreover, carbon-coated nanoparticles are usually in the metallic state, and, compared to the corresponding oxides, they have a higher magnetic moment [25]. In particular, at present, a hot topic is magnetic carbon-coated metal (such as $\mathrm{Fe}, \mathrm{Co}, \mathrm{Ni}$, and $\mathrm{Mn}$ ) composites that are always 
metal phase as the nucleus of core-shell structural composite nanomaterials [26].

In this research study, carbon encapsulated iron nanoparticles (CEINPs) were designed and produced in a way to make them suitable nanocarriers for biomedical applications. So far, different methods including arc discharge [27-29], hydrothermal reaction [30], chemical vapor deposition [31], detonation synthesis [32], chemical vapor condensation [33], magnetron and iron-beam cosputtering [34], laser ablation [35], and cocarbonization [36] are used to synthesize core-shell structures in which metallic nanoparticles are encapsulated by precious metals, polymers, silica, or carbon. However, each of these methods has particular advantages and disadvantages comparing to our method for synthesis of CEINPs. Until now, all reported studies have been only on monoiron nanoparticles and the synthesis of multi-iron core at carbon shell has not yet been reported [37-47].

In current nanoparticle fabrication processes, control of the particle size and composition is still empirical, which means that a large number of experimental trials are required to optimize any given process [48]. Plasma properties such as size, gradient temperature, thermal conductivity, and cooling rate mainly depend on the used inert gases. Consequently, all parameters are kept constant to study the influences of helium and nitrogen on morphologies properties as well as magnetic properties. Here, we present the synthesis of both CEINPs and carbon encapsulated multi-iron nanoparticles. The morphological properties were studied by scanning electron microscopy (SEM) and transmission electron microscopy (TEM). In order to verify the absence of iron oxide, the samples were analyzed by selected area diffraction (SAED), electron energy loss spectroscopy (EELS), and energy-dispersive $\mathrm{X}$-ray spectroscopy (EDX) techniques as well as fast Fourier transform (FFT). In addition, carbon shell crystallinity was investigated by Raman spectroscopy. Afterward, the magnetic properties were obtained from superconducting quantum interference device (SQUID) characterization technique.

\section{Experimental Details}

A modified arc discharge reactor was utilized [49-51]. Nitrogen, helium, and their mixtures were used to synthesize each sample, from $0 \%$ to $100 \%$ of $\mathrm{N}_{2}$. Accordingly, each nanoparticle sample was named based on the nitrogen content; that is, $75 \% \mathrm{~N}+25 \% \mathrm{H}$ is $\mathrm{N} 75$. Two carbon graphite rods (99.99\%) with $\mathrm{mm}$ thickness were prepared as cathode and anode. The cathode electrode was sharpening at an angle near to $20^{\circ}$. Both electrodes were placed in perpendicular position, in order to generate plasma between them. A needle was placed on top of them to deliver the precursor droplets. A spherical glass covered the whole reactor. Then, the precursor was prepared by dissolving ferrocene $(0.5 \mathrm{wt} \%)$ in isooctane. Initially, rotary vane pump was used for making vacuum inside the glass chamber up to a pressure of $1 \mathrm{~Pa}$. Afterward, the gases were introduced inside the glass chamber (continuous flow, $1.61 / \mathrm{min}$ ). The pressure was kept at near atmospheric pressure condition by a micro valve. An arc was generated between anode and cathode by a programmable constant power supply which was set at $40 \mathrm{~A}$ for each production run of $5 \mathrm{~min}$. A syringe pump along with an injector regulates the droplets flow of precursor to the plasma zone (droplet flow rate $1 \mathrm{~mL} / \mathrm{min}$ ). Consequently, the synthesis process took place inside the glass chamber. Afterwards, by using ethanol, the nanoparticles were collected from the glass walls. Finally, nanoparticles were separated from ethanol through a magnetic filtration.

\section{Results and Discussion}

Influences of nitrogen and helium mixtures on CEINPs morphologies and magnetic properties were studied. As it is shown in Figure 1(a), SEM image reflects the CEINPs in spherical and agglomeration form. TEM images in Figures 1(b), 1(c), 1(d), 1(e), and 1(f) show geometry of each individual nanoparticle from N0, N25, N50, N75, and N100 samples, respectively. Spherical iron nanoparticles were observed with mean diameter of $7.5 \mathrm{~nm}$ in N0 samples. By adding $25 \%$ and $50 \%$ nitrogen, mainly oval shape iron nanoparticles with mean dimensions of $7.6 \times 6.5 \mathrm{~nm}$ and $8 \mathrm{~nm} \times 5.6 \mathrm{~nm}$ were obtained in N25 and N50 samples, respectively. Afterwards, by adding $25 \%$ more nitrogen, iron nanoparticles tended to form comparatively smaller oval iron core with mean dimensions of $7.8 \mathrm{~nm} \times 4.7 \mathrm{~nm}$ in N75 sample. Using only nitrogen led to the formation of carbon encapsulated multi-iron nanoparticles (CEMINPs). Consequently, in case of N100 sample, the majority of nanoparticles have small multi-iron cores. CEMINPs mainly consisted of $1 \mathrm{~nm}$ and $2 \mathrm{~nm}$ spherical iron and one or two oval shaped $4.3 \mathrm{~nm}$ $\times 2.5 \mathrm{~nm}$ iron nanoparticles. Figures 2(a) and 2(b) represent TEM images from N100 samples. However, as it is shown in Figure 2(c), iron particles with single oval shape and dimensions of $3 \mathrm{~nm} \times 9 \mathrm{~nm}$ were observed in N100 sample as well. In general, according to the TEM observations as we increased nitrogen gas percentage versus helium to generate plasma, the iron cores were forming oval shape. Based on TEM observations extremely small iron particles of 1 to $3 \mathrm{~nm}$ do not form a bigger single iron core and instead formed multiiron core at carbon shell nanoparticle. Gutsch et al. discussed coalescence effect, which merges nanoparticles to form a bigger one in gas phase production of nanoparticles [52]. The condition of nucleation occurs in iron supersaturation vapor, which permits obtaining the particles with stable growth. Afterwards, coalescence occurs when the concentration is high enough. Once the carbon shell formation begins, the iron cores growth and coalescence are finished. Obtained results highlighted the coalescence effects on formation of iron nanoparticles from sample N100 to N0. The presence of oval iron nanoparticles and multi-iron cores shows the coalescence of the nucleus before starting carbon shell formation. The coalescence of only two iron particles was observed. This can be probably due to surpassing of the critical radius of carbon shell for the growth after the first iron coalescence.

The effect of nitrogen and helium in growth formation can be attributed to their differences in thermal conductivity and plasma temperature. Thermal conductivity of nitrogen is about 9 times smaller than helium. Thermal conductivity regulates the cooling rate and gradient of temperature. 


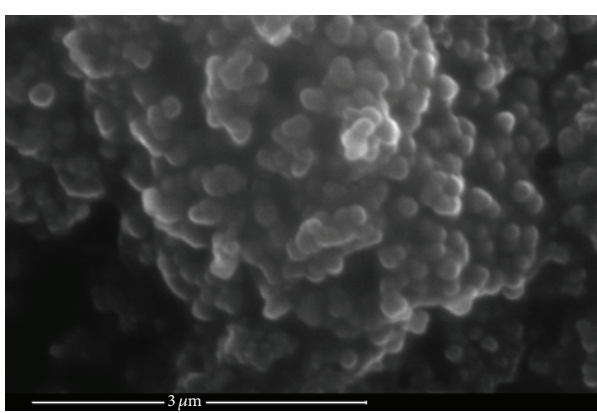

(a)

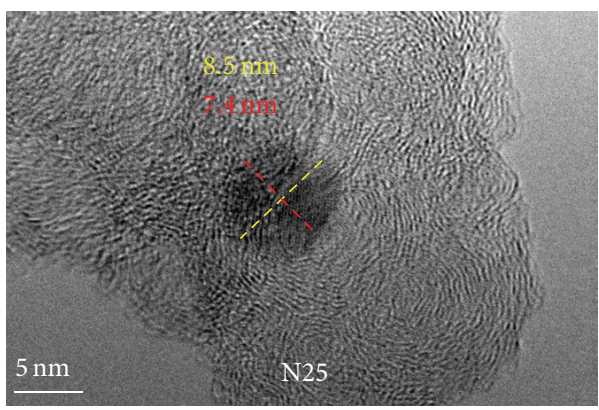

(c)

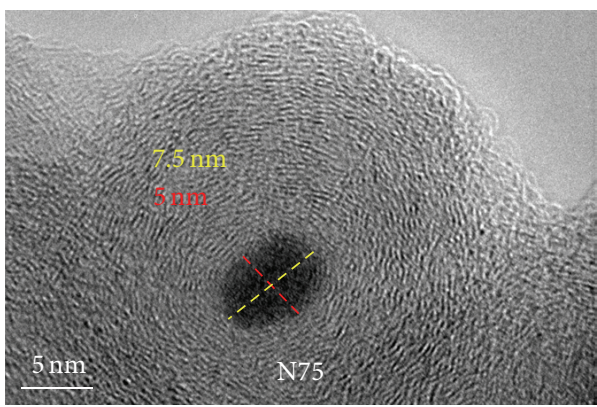

(e)

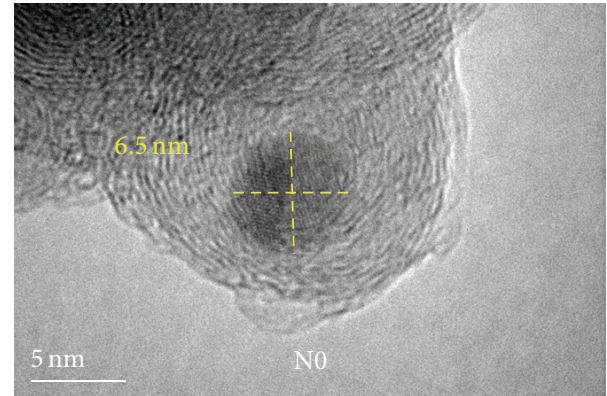

(b)

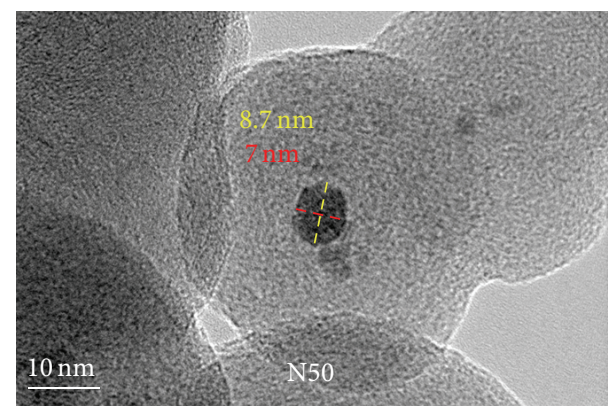

(d)

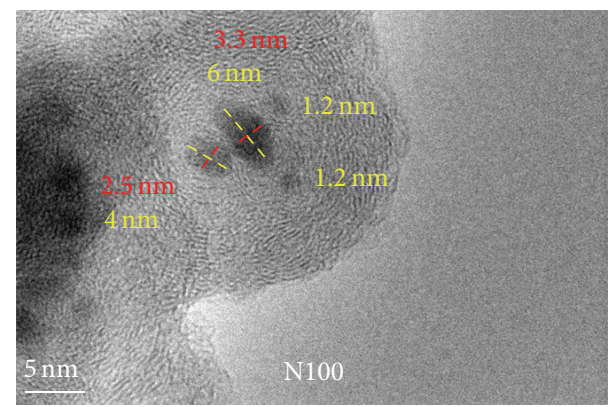

(f)

FIGURE 1: (a) SEM image shows agglomeration of CEINPs produced under nitrogen. (b), (c), (d), (e), and (f) TEM images of CEINPs and CEMINPs are shown from N0, N25, N50, N75, and N100 samples, respectively. It is evident that the morphologies of carbon and iron were changed by increasing nitrogen ratio over helium. The increasing trend in carbon shell formation and the decreasing trend in iron formation are remarkable. Merge and formation of iron nanoparticles from N100 to N0 revealed the role and effect of coalescence in formation of CEINPs and CEMINPs.

Thus comparatively nitrogen exhibits lower cooling rate and longer gradient of temperature over highly supersaturated vapor. Supersaturated vapor results in rapid production of numerous nanoparticles. In addition, relatively more carbon species are available in supersaturated vapor for carbon shell formation at higher temperature generated by nitrogen plasma rather than helium plasma. Accordingly, a decrease trend in carbon shell formation is obvious from sample N100 to N0 in Figure 1. It should be noted that the carbon shell formation limits the critical radius of iron. According to our observation when iron particles are small enough (1-3 nm) they are trapped into a single carbon shell.

Since nitrogen is diatomic gas, its plasma has higher energy contents for a given temperature than the atomic gases such as helium [53]. Because of high electronegativity of nitrogen, more energetic electrons are necessary to produce self-sustained arc discharge. Consequently, when nitrogen is used due to the high temperature the carbon electrodes were deformed during the experiment (Figure S1 in Supplementary Material available online at http://dx.doi.org/10.1155/2015/450183). Regardless of inert gas type, in this arc discharge reactor the electron bombardment begins from cathode to anode and therefore the carbon from the anode electrode was consumed and deformed. This deformation caused changes in plasma shape, made it unstable and prevented continuous synthesis. Moreover, it has negative effect on formation and size distributions of CEINPs and CEMINPs. The size distributions of each sample are shown in Figure S2. N0 sample reveals the best iron size distribution comparing to the other samples, because by using only helium the carbon electrodes show smaller deformation. In spite of usefulness of presented method 


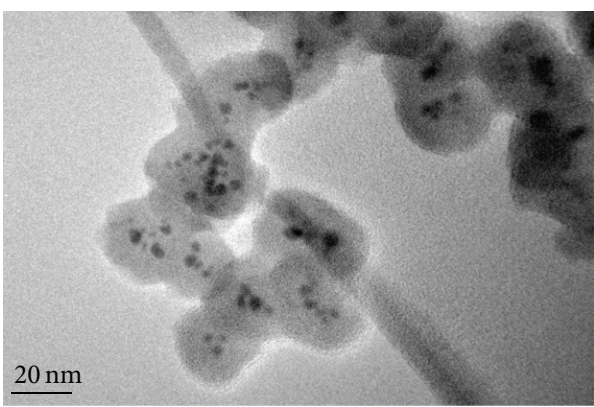

(a)

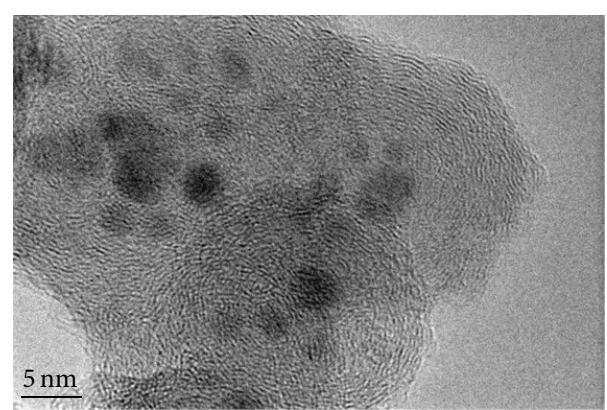

(b)

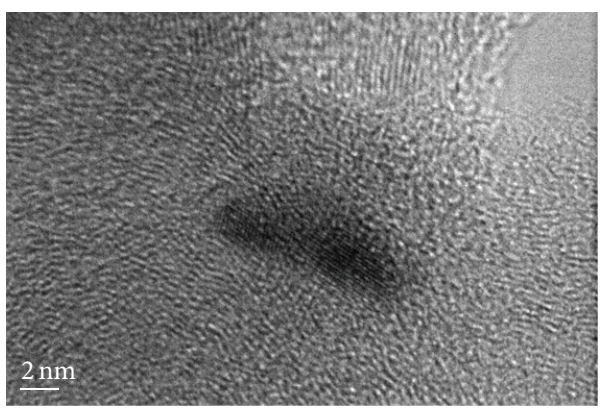

(c)

FIGURE 2: (a) Illustration of CEMINPs; the iron cores are located very close to each other. (b) Close observation of a few individual multiiron cores at carbon shell nanoparticles. (c) Appearance of single oval shape iron nanoparticle in N100 samples; this is probably due to the coalescence between two particles.

for CEINPs and CEMINPs synthesis, plasma instability and discontinuous synthesis are the chief drawbacks. A brief investigation on the deformed carbon electrode by SEM has been done. SEM image from carbon piece taken from anode electrode is presented in Figure 3 and it revealed formation and deposition of spherical carbon particles on top of each other. Spaces between particles are visible clearly; therefore possibly this technique can be used for the synthesis and formation of carbon porous materials. Structure of carbon piece on anode suggests a mechanism of growth, which is large cross section of nanoparticles that facilitates the attachment of free electrons to the nanoparticles in the plasma region, therefore becoming attracted by anode due to the electrostatic forces. This contributes to forming very structured carbon deposition on anode electrode in Figure 3. Thinner carbon deposition on cathode was observed probably due to the neutral nanoparticles and negative radicals of carbon. Moreover, the cathode suffers a significant ion bombardment (e.g., $\mathrm{CH}_{3}{ }^{+}$ [54]). In addition, according to the given growth mechanism of CEINPs, the nitrogen plasma has higher temperature compared to helium plasma, which results in higher availability of carbon species. According to our observation, higher contribution of nitrogen shows an increase in deposition of carbon related structure on the electrodes.

Due to the importance of iron core sufficient protection and in order to show the efficiency of carbon shells against the oxidation of core, samples were evaluated by SAED and FFT techniques. SAED is shown in Figure 4(a); diffraction points of iron are indexed to $\alpha$-Fe crystal structure as identified by diffraction rings corresponding to the $(-1,0,1)$ and

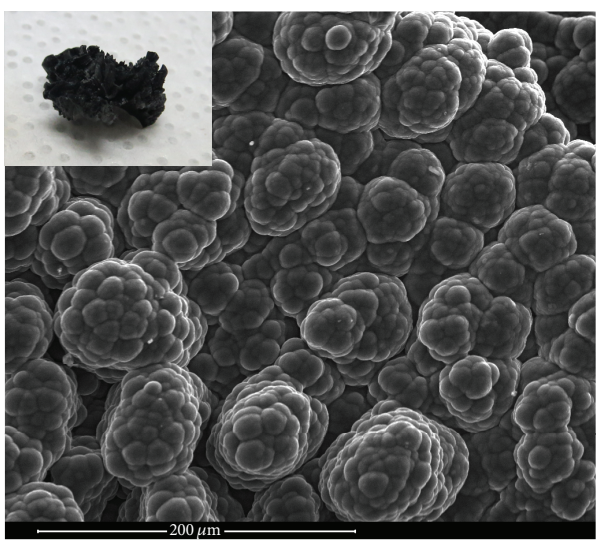

FIgURE 3: SEM image of CEINPs deposited on anode electrode when nitrogen plasma is used. The inset image is the carbon piece in question. Carbon spherical formation and the pores between them are clearly visible. Possibly nanopores also exist but cannot be observed at this resolution.

$(0,-1,1)$ planes. However, as it is shown in Figure 4(b) from FFT analysis, trace of iron carbide was detected in interface between core and shell. Most importantly, diffraction points of iron oxides were not detected in any of our samples. In addition, nanoparticles were analyzed by EELS and EDX analysis along with chemical mapping. Figure 5 represents an example of EELS analysis at nanoscale and EDX together with chemical mapping at micro scale. As a result, absences of oxygen are confirmed in iron cores; therefore carbon shell 


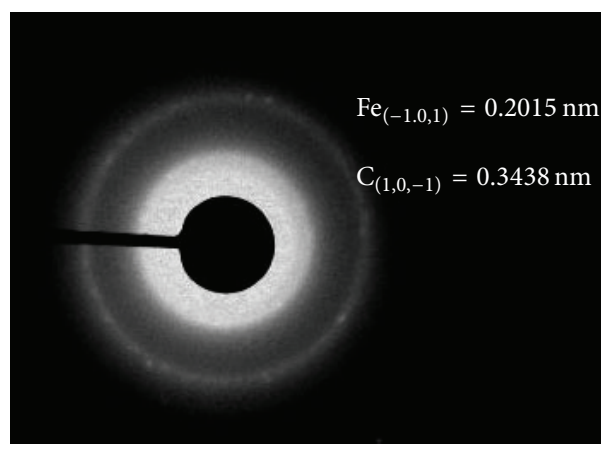

(a)

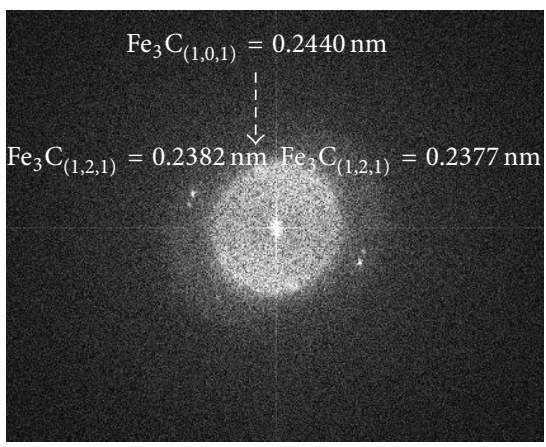

(b)

FIGURE 4: (a) SAED image reveals diffuse rings due to the presence of both nanocrystalline and amorphous phases. (b) FFT analysis shows trace of iron carbide.

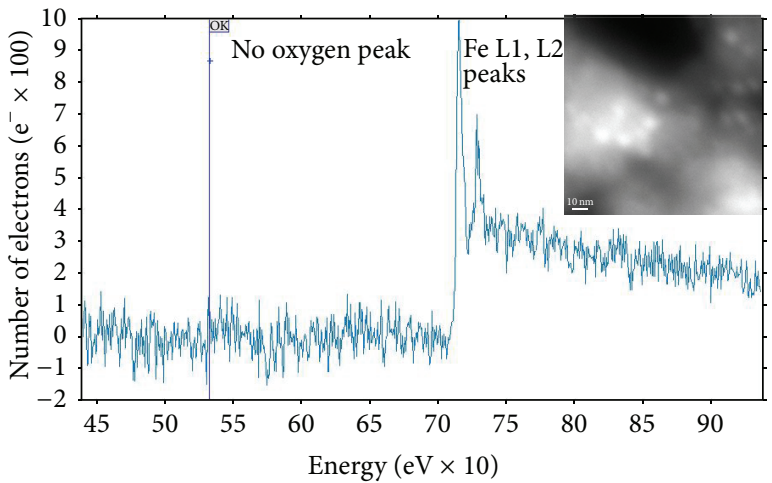

(a)

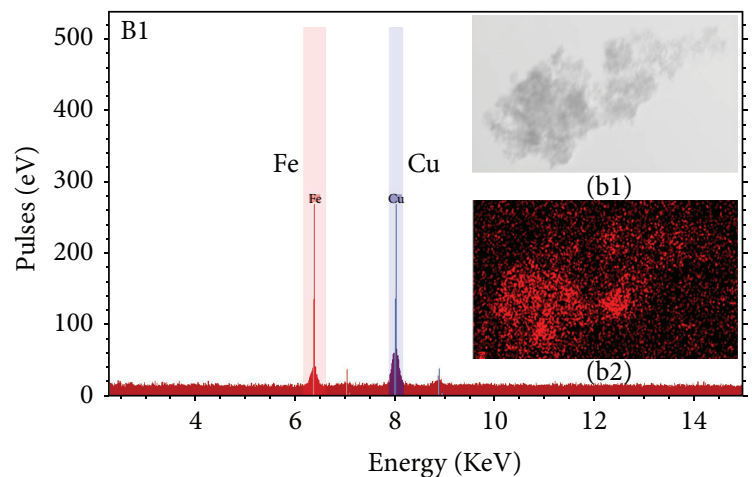

(b)

FIGURE 5: (a) Spectra of EELS analysis at nanoscale and associated TEM image are in the inset; EELS analysis shows no oxygen peak. (b) EDX analysis at microscale shows only Fe peak without oxygen peak as well (Cu peak is due to the grid). EDX associated TEM image is in (b1) inset, and associated chemical mapping is in (b2) inset; Red spots represent Fe.

properly sealed the iron cores in all samples. In addition, the fewer defects in carbon shell provide higher protection efficiency. Accordingly, carbon shells order/disorder structure was analyzed by RAMAN spectroscopy. The D-band of metal/C nanoparticles is considered to be a disorder-induced feature owing to lattice distortion or amorphous carbon background signal [55]. The peak intensity ratio $I_{D} / I_{G}$ is often used to determine the extent of structural disorder in graphite and/or the size of the graphitic domains [56]. Thus, the decoupled $I_{D} / I_{G}$ are considered. $D$ and $G$ peaks are located near $\sim 1350$ and $\sim 1600 \mathrm{~cm}^{-1}$, respectively. RAMAN spectra peak-height ratios $I_{D} / I_{G}$ were determined from Lorentzian fittings as the example spectra shown in Figure 6(a). The carbon shells crystallinities degree $\left(I_{D} / I_{G}\right)$ was obtained for each sample accordingly and was less than one for all samples, indicating high carbon crystallinity. Comparison of $I_{D} / I_{G}$ of each sample in Figure 6(b) reveals slight decrease in $I_{D} / I_{G}$ ratio as more nitrogen is used. This means that the graphitization degree of the samples increases slightly with increasing nitrogen gas concentration. Zhao et al. studied carbon nanostructures production by AC arc discharge plasma process at atmospheric pressure and concluded that the value of $I_{D} / I_{G}$ decreases from 1.2 to 0.6 with the increasing arc discharge current [57]. In their study, the temperature has been increased by increasing arc discharge electric current, while in our study the electric current was kept constant and the temperature was regulated according to the gas mixture of $\mathrm{N} 2 / \mathrm{He}$ at near atmospheric pressure. Nitrogen exhibits higher plasma temperature in comparison with helium plasma; the lowest $I_{D} / I_{G}$ corresponds to N100 sample $\left(I_{D} / I_{G} \sim 0.81\right)$, when only nitrogen is used.

Afterwards, the magnetic properties were characterized by superconducting quantum interference device (SQUID). CEINPs and CEMINPs magnetic behaviors were investigated at $300 \mathrm{~K}$. Figure 7 (a) represents comparison of normalized hysteresis curve for N100, N75, N50, N25, and N0 samples. The saturation magnetization for the nanocrystalline ferrites, in general, is found to be lower compared to their bulk value, which is attributed to surface spin effects [58]. Saturation magnetization largely depends on size, shape, metal composition, and crystalline magnetic anisotropy energy, as well as coating by nonmagnetic materials $[52,59]$. N0 sample exhibits higher saturation magnetization than samples generated in presence of nitrogen. Therefore, from morphological point of view, as the iron cores are reforming from spherical shape to smaller oval shape, the saturation magnetization is 


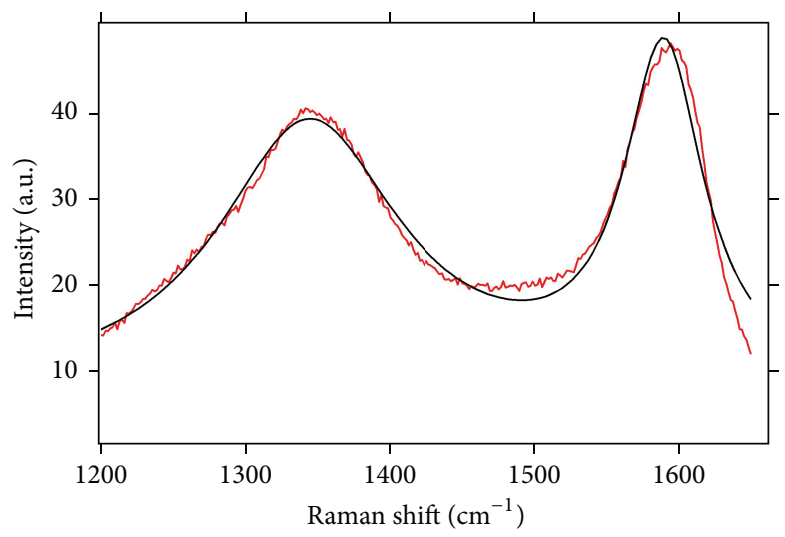

(a)

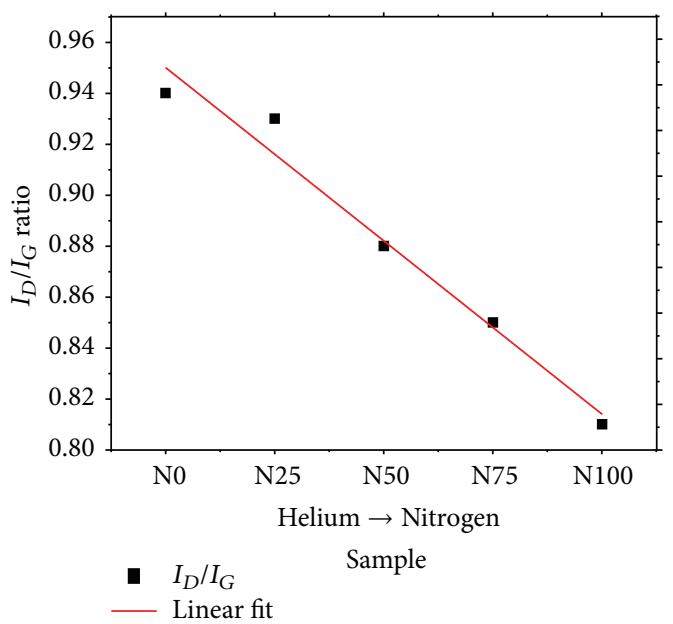

(b)

Figure 6: (a) Micro-Raman spectra of N100 sample fitted to Lorentzian function; $D$ and $G$ peaks are located near $\sim 1350$ and $\sim 1600 \mathrm{~cm}^{1}$. (b) This graph shows comparison between $I_{D} / I_{G}$ ratios for each sample. By using only helium (N0), the number of defects is always higher than samples that were obtained by using nitrogen and/or its mixture with helium.

decreasing. Size dependency of saturation magnetization is reported in the literature [60-62]. Interestingly, in spite of smaller iron cores, the multi-iron cores nanoparticles (N100 sample) exhibit higher saturation magnetization than single oval shape nanoparticles in N75 sample. Jafari et al. studied the effect of carbon shell on the structural and magnetic properties of $\mathrm{Fe}_{3} \mathrm{O}_{4}$ and concluded that the magnetization of $\mathrm{Fe}_{3} \mathrm{O}_{4}$ nanoparticles was reduced after coating with carbon [63]. Bittova et al. carried out a research study on the effects of coating on dipolar interparticle interactions and showed the reduction of saturation magnetization after coating as well [64]. In our samples, the single iron cores are mainly located in the center of a large carbon shell while the multiiron cores are distributed in a carbon shell; therefore probably carbon shell has less negative magnetic effect to multi-iron cores comparatively due to higher average distance between center iron cores. Increasing the saturation magnetization of magnetic nanoparticles may permit more effective development of multifunctional agents for simultaneous targeted cell delivery, magnetic resonance imaging contrast enhancement, and targeted cancer therapy in the form of local hyperthermia [65]. Zero-field-cooling (ZFC) and field-cooling (FC) curves exhibit the main attribute of superparamagnetic system. ZFC and FC magnetization from N100 sample are plotted in Figure 7(b). All the samples were in superparamagnetic state at room temperature. Multi-iron cores nanoparticles blocking temperature $\left(T_{B}\right)$ value $(30 \mathrm{~K})$ is lowest among the others, followed by N75, N50 $43 \mathrm{~K}, \mathrm{~N} 25$, and N0 with $36 \mathrm{~K}, 43 \mathrm{~K}$, $45 \mathrm{~K}$, and $55 \mathrm{~K}$, respectively. The $T_{B}$ values versus median size from size distribution analysis are plotted in Figure $7(\mathrm{c})$. The finding that $T_{B}$ decreases as the size of the nanoparticles decreases is consistent with conventional Stoner-Wohlfarth theory [66]: the energy barrier, over which the magnetization of the nanoparticles should be thermally activated, increases as the size of the nanoparticles increases. Consequently, the lowest $T_{B}$ value of CEMINPs confirms the existence of smaller iron nanoparticles comparing to other samples. The sum of multi-iron cores in each carbon shell is greater than a single oval shape in N75 sample but its $T_{B}$ value is lower. This is an interesting characteristic of CEMINPs. The result of low $T_{B}$ value opens the possibility of using superparamagnetic particles in applications that required high standard and needs to operate at very low temperatures such as sensors in aerospace industry. Moreover, magnetic properties results of presented CEMINPs and CEINPs are important because in principle interests in magnetic particles are all due to the possibility to governing, heating, and detecting them by external magnetic field for biomedical applications.

\section{Conclusion}

Mono- and multi-iron nanoparticles encapsulated in carbon shell were controllably synthesized using a new modified arc discharge reactor. Influence of nitrogen on plasma evidenced higher concentration of carbon species and changes the morphologies of CEINPs and CEMINPs. Moreover, the nature of gas has influences on yield of nanoparticles; in particular, nitrogen plasma provided highest yield in our study. The diffraction points of iron oxide were not detected from SAED and FFT techniques. In addition, EELS analysis at nanoscale and EDX analysis at microscale showed no trace of oxygen in iron cores; hence samples were well protected by carbon shells. Based on the Raman spectroscopy results, best carbon crystallinity is observed when only nitrogen is used. Sufficient iron core protection along with low defects of carbon shell structure promotes very small pores and therefore minimizes the possibility of iron oxidation, degradation, and/or possible toxicity; hence both CEINPs and CEMINPs are suitable for highly chemically active environment. All the particles show a superparamagnetic behavior at room temperature as determined by SQUID measurements. Lowest blocking temperature of $30 \mathrm{~K}$ evidenced the synthesis of smaller multicores 


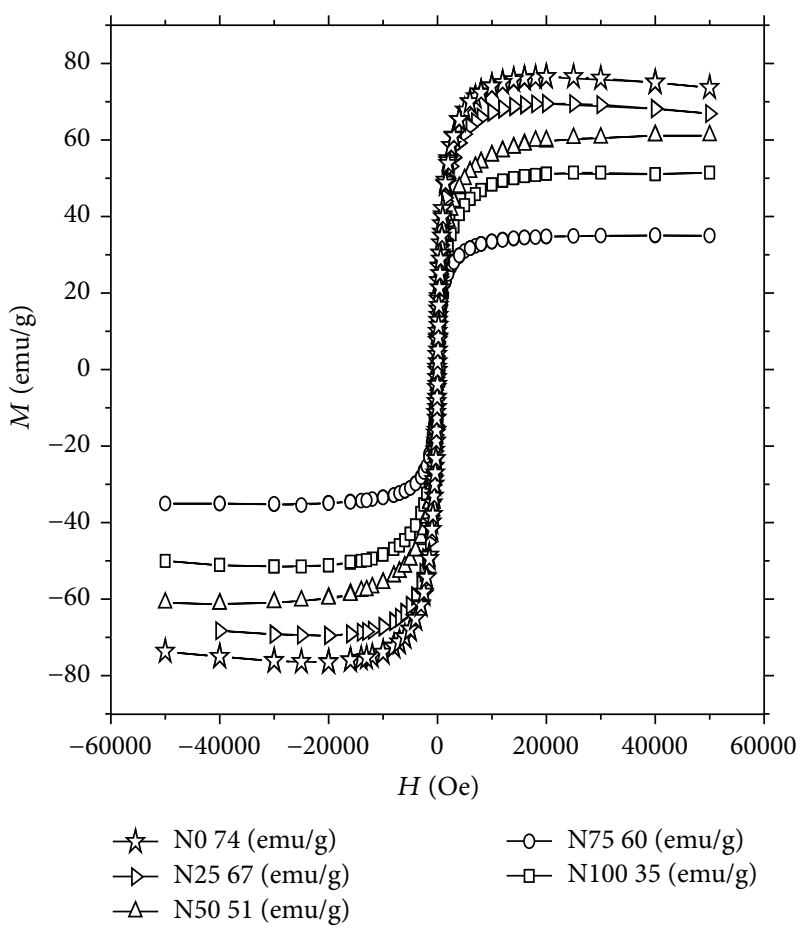

(a)

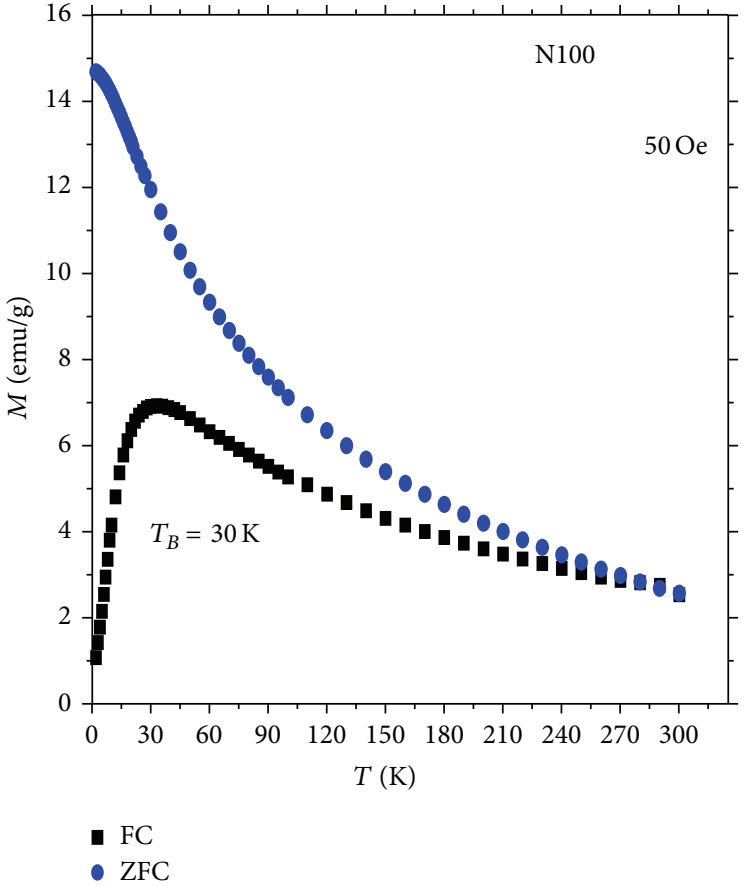

(b)

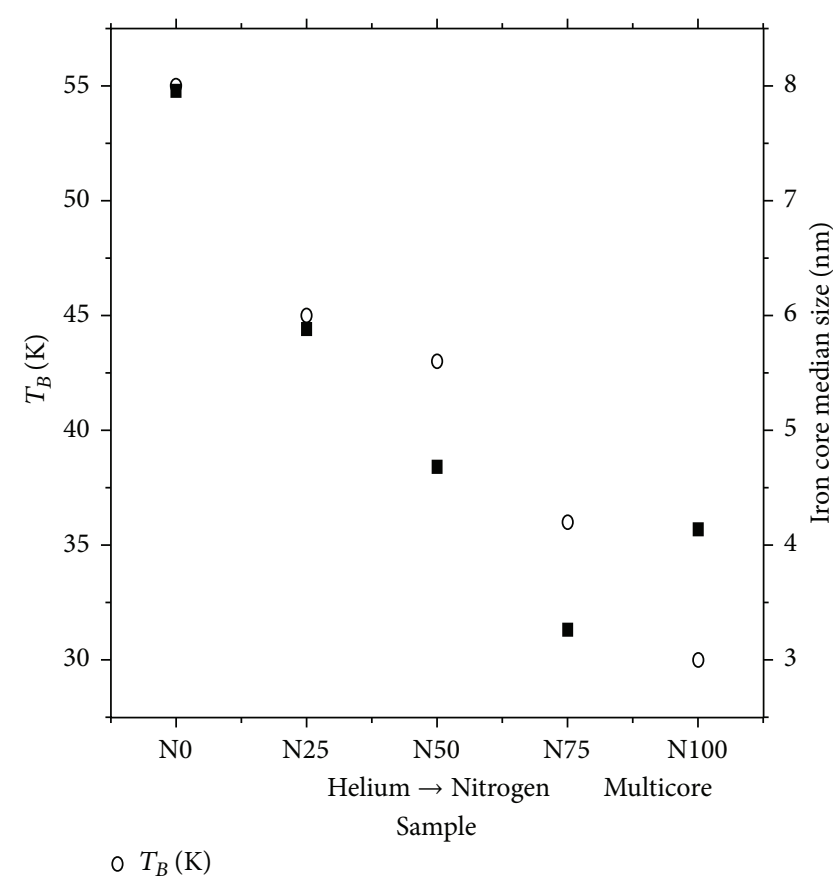

- Iron core median size $(\mathrm{nm})$

(c)

Figure 7: (a) Hysteresis curve comparison of N0, N25, N50, N75, and N100 samples and the saturation magnetization are 74, 67, 60, 35, and 51 (emu/g), respectively. (b) Zero-field-cooled and field-cooled magnetization curve of multi-iron core nanoparticles measured in 50 Oe. This type of nanoparticles exhibited low blocking temperature $(30 \mathrm{~K})$ and is in superparamagnetic state above $240 \mathrm{~K}$. (c) This graph shows the changes between blocking temperature and iron median size for each sample. 
than monocore nanoparticles. Interestingly, although multiiron cores are smaller than iron core in CEINPs (N75), they exhibit higher saturation magnetization; one of the reasons can be the existence of a few iron cores in a single carbon shell and therefore carbon shell has lower negative effect on magnetic properties. Owing to the extremely close distance (around $2 \mathrm{~nm}$ ) between iron cores in CEMINPs, there will be higher visibility comparing to CEINPs and therefore this type of nanoparticles has more priority to be used as contrast agent. On the other hand it is noteworthy to mention that, due to the carbon shell spherical shape and iron superparamagnetic behavior, their movement in body fluid can be smooth and controllable and hence the CEINPs can be potentially used in drug delivery. It is concluded that the gas nature of the reactor plasma used in the research project has significant effect on the morphological properties of CEINPs. Accordingly, by this method, CEINPs and CEMINPs can be synthesized based on the desired applications.

\section{Conflict of Interests}

The authors declare that there is no conflict of interests regarding the publication of this paper.

\section{Acknowledgments}

The authors acknowledge Centres Científics i Tecnològics of Universitat de Barcelona (CCIT-UB). This work was partially financed by the MICYN of Spanish Government, under Contract MAT2010 20468, and by AGAUR of Generalitat de Catalunya, under Contracts 2009SGR00185 and 2014SGR0948.

\section{References}

[1] G. Reiss and A. Hütten, "Magnetic nanoparticles: applications beyond data storage," Nature Materials, vol. 4, no. 10, pp. 725726, 2005.

[2] Z. Yang and S. Wang, "High cycling performance cathode material: interconnected $\mathrm{LiFePO}_{4}$ /carbon nanoparticles fabricated by sol-gel method," Journal of Nanomaterials, vol. 2014, Article ID 801562, 7 pages, 2014.

[3] L.-G. Zamfir, I. Geana, S. Bourigua et al., "Highly sensitive labelfree immunosensor for ochratoxin A based on functionalized magnetic nanoparticles and EIS/SPR detection," Sensors and Actuators B: Chemical, vol. 159, no. 1, pp. 178-184, 2011.

[4] S. Singamaneni, V. N. Bliznyuk, C. Binek, and E. Y. Tsymbal, "Magnetic nanoparticles: recent advances in synthesis, selfassembly and applications," Journal of Materials Chemistry, vol. 21, no. 42, pp. 16819-16845, 2011.

[5] M. Colombo, S. Carregal-Romero, M. F. Casula et al., "Biological applications of magnetic nanoparticles," Chemical Society Reviews, vol. 41, no. 11, pp. 4306-4334, 2012.

[6] M. Ishii, R. Shibata, Y. Numaguchi et al., "Enhanced angiogenesis by transplantation of mesenchymal stem cell sheet created by a novel magnetic tissue engineering method," Arteriosclerosis, Thrombosis, and Vascular Biology, vol. 31, no. 10, pp. 2210-2215, 2011.

[7] M. Cao, Z. Li, J. Wang et al., "Food related applications of magnetic iron oxide nanoparticles: enzyme immobilization, protein purification, and food analysis," Trends in Food Science \& Technology, vol. 27, no. 1, pp. 47-56, 2012.

[8] O. Veiseh, J. W. Gunn, and M. Zhang, "Design and fabrication of magnetic nanoparticles for targeted drug delivery and imaging," Advanced Drug Delivery Reviews, vol. 62, no. 3, pp. 284-304, 2010.

[9] S. Yanase, J. Nomura, Y. Matsumura, H. Kato, and T. Tagawa, "Hyperthermia enhances the antitumor effect of photodynamic therapy with ALA hexyl ester in a squamous cell carcinoma tumor model," Photodiagnosis and Photodynamic Therapy, vol. 9, no. 4, pp. 369-375, 2012.

[10] H. B. Na, I. C. Song, and T. Hyeon, "Inorganic nanoparticles for MRI contrast agents," Advanced Materials, vol. 21, no. 21, pp. 2133-2148, 2009.

[11] A. Ruiz, G. Salas, M. Calero et al., "Short-chain PEG molecules strongly bound to magnetic nanoparticle for MRI long circulating agents," Acta Biomaterialia, vol. 9, no. 5, pp. 6421-6430, 2013.

[12] R. D. Ambashta and M. Sillanpää, "Water purification using magnetic assistance: a review," Journal of Hazardous Materials, vol. 180, no. 1-3, pp. 38-49, 2010.

[13] X. Zuo, C. Peng, Q. Huang et al., "Design of a carbon nanotube/magnetic nanoparticle-based peroxidase-like nanocomplex and its application for highly efficient catalytic oxidation of phenols," Nano Research, vol. 2, no. 8, pp. 617-623, 2009.

[14] S. C. Tsang, V. Caps, I. Paraskevas, D. Chadwick, and D. Thompsett, "Magnetically separable, carbon-supported nanocatalysts for the manufacture of fine chemicals," Angewandte Chemie, vol. 43, no. 42, pp. 5645-5649, 2004.

[15] M. Bystrzejewski, R. Klingeler, T. Gemming, B. Büchner, and M. H. Rümmeli, "Synthesis of carbon-encapsulated iron nanoparticles by pyrolysis of iron citrate and poly(vinyl alcohol): a critical evaluation of yield and selectivity," Nanotechnology, vol. 22, no. 31, Article ID 315606, 2011.

[16] A. G. Roca, D. Carmona, N. Miguel-Sancho et al., "Surface functionalization for tailoring the aggregation and magnetic behaviour of silica-coated iron oxide nanostructures," Nanotechnology, vol. 23, no. 15, Article ID 155603, 2012.

[17] A. M. Nowicka, A. Kowalczyk, M. Bystrzejewski, M. Donten, and Z. Stojek, "Carbon-encapsulated iron nanoparticles used to generate magnetic field and to enhance substrate transport at electrode surface," Electrochemistry Communications, vol. 20, no. 1, pp. 4-6, 2012.

[18] M. Reza Sanaee and E. Bertran, "Synthesis of carbon encapsulated iron nanoparticles for applications in biomedicine," Journal of Nanomedicine and Nanotechnology, vol. 4, no. 6, p. 219, 2013.

[19] M. Reza Sanaee, V. M. Freire, N. Aguil, E. Bertran, and N. Aguiló-Aguayo, "Design and synthesis of carbon encapsulated iron nanoparticle for drug delivery," in Proceedings of the 10th International Conference on Nano Bio and Med, pp. 212-213, Phantoms Foundation, Bilbao, Spain, 2013.

[20] K. Wang, Y. Huang, T. Han, Y. Zhao, H. Huang, and L. Xue, "Facile synthesis and performance of polypyrrole-coated hollow $\mathrm{Zn}_{2} \mathrm{SnO}_{4}$ boxes as anode materials for lithium-ion batteries," Ceramics International, vol. 40, no. 1, pp. 2359-2364, 2014.

[21] W. Schärtl, "Current directions in core-shell nanoparticle design," Nanoscale, vol. 2, no. 6, pp. 829-843, 2010.

[22] P. Wu, N. Du, H. Zhang, J. Yu, and D. Yang, "Carbon nanocapsules as nanoreactors for controllable synthesis of encapsulated iron and iron oxides: magnetic properties and reversible lithium 
storage," The Journal of Physical Chemistry C, vol. 115, no. 9, pp. 3612-3620, 2011.

[23] F. Avalos-Belmontes, L. F. Ramos-Devalle, E. Ramírez-Vargas, S. Sánchez-Valdes, J. Méndez-Nonel, and R. Zitzumbo-Guzmán, "Nucleating effect of carbon nanoparticles and their influence on the thermal and chemical stability of polypropylene," Journal of Nanomaterials, vol. 2012, Article ID 406214, 8 pages, 2012.

[24] Y. R. Uhm and C. K. Rhee, "Synthesis and magnetic properties of $\mathrm{Ni}$ and carbon coated $\mathrm{Ni}$ by levitational gas condensation (LGC)," Journal of Nanomaterials, vol. 2013, Article ID 427489, 6 pages, 2013.

[25] Z. Chen, G. Hong, H. Wang et al., "Graphite-coated magnetic nanoparticle microarray for few-cells enrichment and detection," ACS Nano, vol. 6, no. 2, pp. 1094-1101, 2012.

[26] N. Luo, K. Liu, Z. Liu et al., "Controllable synthesis of carbon coated iron-based composite nanoparticles," Nanotechnology, vol. 23, no. 47, Article ID 475603, 2012.

[27] "Study of carbon encapsulated iron nanoparticles produced by a modified arc discharge by applying nitrogen, argon and helium," in Proceedings of the International Conference on Nanoscience and Nanotechnology, pp. 160-161, Phantoms Foundation, Bilbao, Spain, 2013.

[28] M. R. Sanaee, N. Aguiló-Aguayo, and E. Bertran, "Influences of argon-helium mixtures on the carbon-coated iron nanoparticles produced by a modified arc discharge," in Proceedings of the 9th International Conference on Nanoscience and Nanotechnologies, p. 12, Artion, Thessaloniki, Greece, 2012.

[29] L. Shen and N. Wang, "Effect of nitrogen pressure on the structure of Cr-N, Ta-N, Mo-N, and W-N nanocrystals synthesized by arc discharge," Journal of Nanomaterials, vol. 2011, Article ID 781935, 5 pages, 2011.

[30] S. Zhang, H. Niu, Z. Hu, Y. Cai, and Y. Shi, "Preparation of carbon coated $\mathrm{Fe}_{3} \mathrm{O}_{4}$ nanoparticles and their application for solid-phase extraction of polycyclic aromatic hydrocarbons from environmental water samples," Journal of Chromatography A, vol. 1217, no. 29, pp. 4757-4764, 2010.

[31] H. Cao, G. Huang, S. Xuan, Q. Wu, F. Gu, and C. Li, "Synthesis and characterization of carbon-coated iron core/shell nanostructures," Journal of Alloys and Compounds, vol. 448, no. 1-2, pp. 272-276, 2008.

[32] Y. Lu, Z. Zhu, and Z. Liu, "Carbon-encapsulated Fe nanoparticles from detonation-induced pyrolysis of ferrocene," Carbon, vol. 43, no. 2, pp. 369-374, 2005.

[33] Z. H. Wang, Z. D. Zhang, C. J. Choi, and B. K. Kim, "Structure and magnetic properties of $\mathrm{Fe}(\mathrm{C})$ and $\mathrm{Co}(\mathrm{C})$ nanocapsules prepared by chemical vapor condensation," Journal of Alloys and Compounds, vol. 361, no. 1-2, pp. 289-293, 2003.

[34] J. Nishijo, C. Okabe, J. Bushiri, K. Kosugi, N. Nishi, and H. Sawa, "Formation of carbon-encapsulated metallic nanoparticles from metal acetylides by electron beam irradiation," The European Physical Journal D-Atomic, Molecular, Optical and Plasma Physics, vol. 34, no. 1-3, pp. 219-222, 2005.

[35] J. B. Park, S. H. Jeong, M. S. Jeong, J. Y. Kim, and B. K. Cho, "Synthesis of carbon-encapsulated magnetic nanoparticles by pulsed laser irradiation of solution," Carbon, vol. 46, no. 11, pp. 1369-1377, 2008.

[36] J. Huo, H. Song, and X. Chen, "Preparation of carbonencapsulated iron nanoparticles by co-carbonization of aromatic heavy oil and ferrocene," Carbon, vol. 42, no. 15, pp. 31773182, 2004.
[37] R. P. Chaudhary, S. K. Mohanty, and A. R. Koymen, "Novel method for synthesis of Fe core and C shell magnetic nanoparticles," Carbon, vol. 79, pp. 67-73, 2014.

[38] M. Zhao, H. Song, X. Chen, and W. Lian, "Large-scale synthesis of onion-like carbon nanoparticles by carbonization of phenolic resin," Acta Materialia, vol. 55, no. 18, pp. 6144-6150, 2007.

[39] N. Luo, X. Li, X. Wang, H. Yan, C. Zhang, and H. Wang, "Synthesis and characterization of carbon-encapsulated iron/iron carbide nanoparticles by a detonation method," Carbon, vol. 48, no. 13, pp. 3858-3863, 2010.

[40] M. Bystrzejewski, "Synthesis of carbon-encapsulated iron nanoparticles via solid state reduction of iron oxide nanoparticles," Journal of Solid State Chemistry, vol. 184, no. 6, pp. 14921498, 2011.

[41] Y. V. Fedoseeva, L. G. Bulusheva, A. V. Okotrub et al., "Effect of oxidation and heat treatment on the morphology and electronic structure of carbon-encapsulated iron carbide nanoparticles," Materials Chemistry and Physics, vol. 135, no. 1, pp. 235-240, 2012.

[42] Z. Abdullaeva, E. Omurzak, C. Iwamoto et al., "Onion-like carbon-encapsulated $\mathrm{Co}, \mathrm{Ni}$, and $\mathrm{Fe}$ magnetic nanoparticles with low cytotoxicity synthesized by a pulsed plasma in a liquid," Carbon, vol. 50, no. 5, pp. 1776-1785, 2012.

[43] V. Gupta, M. K. Patra, A. Shukla et al., "Synthesis of core-shell iron nanoparticles from decomposition of $\mathrm{Fe}-\mathrm{Sn}$ nanocomposite and studies on their microwave absorption properties," Journal of Nanoparticle Research, vol. 14, no. 12, article no. 1271, 2012.

[44] M. Sharma, S. Mantri, and D. Bahadur, "Study of carbon encapsulated iron oxide/iron carbide nanocomposite for hyperthermia," Journal of Magnetism and Magnetic Materials, vol. 324, no. 23, pp. 3975-3980, 2012.

[45] H. Zhang, C. Liang, J. Liu, Z. Tian, and G. Shao, “The formation of onion-like carbon-encapsulated cobalt carbide core/shell nanoparticles by the laser ablation of metallic cobalt in acetone," Carbon, vol. 55, pp. 108-115, 2013.

[46] O. Łabędź, A. Grabias, W. Kaszuwara, and M. Bystrzejewski, "Influence of $\mathrm{Al}$ on synthesis and properties of carbonencapsulated iron nanoparticles," Journal of Alloys and Compounds, vol. 603, pp. 230-238, 2014.

[47] A. Mostofizadeh, Y. Li, B. Song, and Y. Huang, "Synthesis, properties, and applications of low-dimensional carbon-related nanomaterials," Journal of Nanomaterials, vol. 2011, Article ID 685081, 21 pages, 2011.

[48] M. Shigeta and A. B. Murphy, "Thermal plasmas for nanofabrication," Journal of Physics D: Applied Physics, vol. 44, no. 17, Article ID 174025, 2011.

[49] E. Bertran-Serra and M. J. Inestrosa-Izurieta, "Method and reactor for the production of carbon-coated nanoparticles," Patent, Barcelona, Spain, 2012.

[50] M. R. Sanaee, O. Arteaga, and E. Bertran, "Influence of plasma reactor parameters on carbon coating of iron nanoparticle," in Proceedings of the International Conference on Nanoscience and Nanotechnology, p. 9, Phantoms Foundation, Madrid, Spain, 2014.

[51] N. Aguiló-Aguayo, Z. Liu, E. Bertran, and J. Yang, “Thermalinduced structural evolution of carbon-encapsulated iron nanoparticles generated by two different methods," The Journal of Physical Chemistry C, vol. 117, no. 37, pp. 19167-19174, 2013.

[52] A. Gutsch, M. Krämer, G. Michael, H. Mühlenweg, M. Pridöhl, and G. Zimmermann, "Gas-phase production of nanoparticles," KONA Powder and Particle Journal, vol. 20, pp. 24-37, 2002. 
[53] A. D. Gerdeman, Arc Plasma Technology in Materials Science, Springer, Berlin, Germany, 1972.

[54] B. F. Gordiets, M. J. Inestrosa-Izurieta, A. Navarro, and E. Bertran, "Nanoparticles in SiH4-Ar plasma: modelling and comparison with experimental data," Journal of Applied Physics, vol. 110, no. 10, Article ID 103302, 2011.

[55] S. J. Lee, J. Jung, M. A. Kim, Y.-R. Kim, and J. K. Park, "Synthesis of highly stable graphite-encapsulated metal (Fe, Co, and $\mathrm{Ni}$ ) nanoparticles," Journal of Materials Science, vol. 47, no. 23, pp. 8112-8117, 2012.

[56] V. A. Sethuraman, L. J. Hardwick, V. Srinivasan, and R. Kostecki, "Surface structural disordering in graphite upon lithium intercalation/deintercalation," Journal of Power Sources, vol. 195, no. 11, pp. 3655-3660, 2010.

[57] S. Zhao, R. Hong, Z. Luo, H. Lu, and B. Yan, "Carbon nanostructures production by AC arc discharge plasma process at atmospheric pressure," Journal of Nanomaterials, vol. 2011, Article ID 346206, 6 pages, 2011.

[58] S. S. Kader, D. P. Paul, and S. M. Hoque, "Effect of temperature on the structural and magnetic properties of $\mathrm{CuFe}_{2} \mathrm{O}_{4}$ nano particle prepared by chemical co-precipitation method," International Journal of Materials, Mechanics and Manufacturing, vol. 2, no. 1, pp. 5-8, 2014.

[59] T. Sato, T. Iijima, M. Seki, and N. Inagaki, "Magnetic properties of ultrafine ferrite particles," Journal of Magnetism and Magnetic Materials, vol. 65, no. 2-3, pp. 252-256, 1987.

[60] P. Dutta, S. Pal, M. S. Seehra, N. Shah, and G. P. Huffman, "Size dependence of magnetic parameters and surface disorder in magnetite nanoparticles," Journal of Applied Physics, vol. 105, no. 7, Article ID 07B501, 2009.

[61] R. D. Sánchez, J. Rivas, P. Vaqueiro, M. A. López-Quintela, and D. Caeiro, "Particle size effects on magnetic properties of yttrium iron garnets prepared by a sol-gel method," Journal of Magnetism and Magnetic Materials, vol. 247, no. 1, pp. 92-98, 2002.

[62] M. S. Seehra, V. Singh, P. Dutta et al., "Size-dependent magnetic parameters of fcc FePt nanoparticles: applications to magnetic hyperthermia," Journal of Physics D: Applied Physics, vol. 43, no. 14, Article ID 145002, 2010.

[63] A. Jafari, K. Boustani, and S. F. Shayesteh, "Effect of carbon shell on the structural and magnetic properties of $\mathrm{Fe}_{3} \mathrm{O}_{4}$ superparamagnetic nanoparticles," Journal of Superconductivity and Novel Magnetism, vol. 27, no. 1, pp. 187-194, 2014.

[64] B. Bittova, J. Poltierova-Vejpravova, A. Roca, M. Morales, and V. Tyrpekl, "Effects of coating on magnetic properties in iron oxide nanoparticles," Journal of Physics: Conference Series, vol. 200, Article ID 072012, 2010.

[65] C. G. Hadjipanayis, M. J. Bonder, S. Balakrishnan, X. Wang, H. Mao, and G. C. Hadjipanayis, "Metallic iron nanoparticles for MRI contrast enhancement and local hyperthermia," Small, vol. 4, no. 11, pp. 1925-1929, 2008.

[66] Y. Lee, J. Lee, C. J. Bae et al., "Large-scale synthesis of uniform and crystalline magnetite nanoparticles using reverse micelles as nanoreactors under reflux conditions," Advanced Functional Materials, vol. 15, no. 3, pp. 503-509, 2005. 

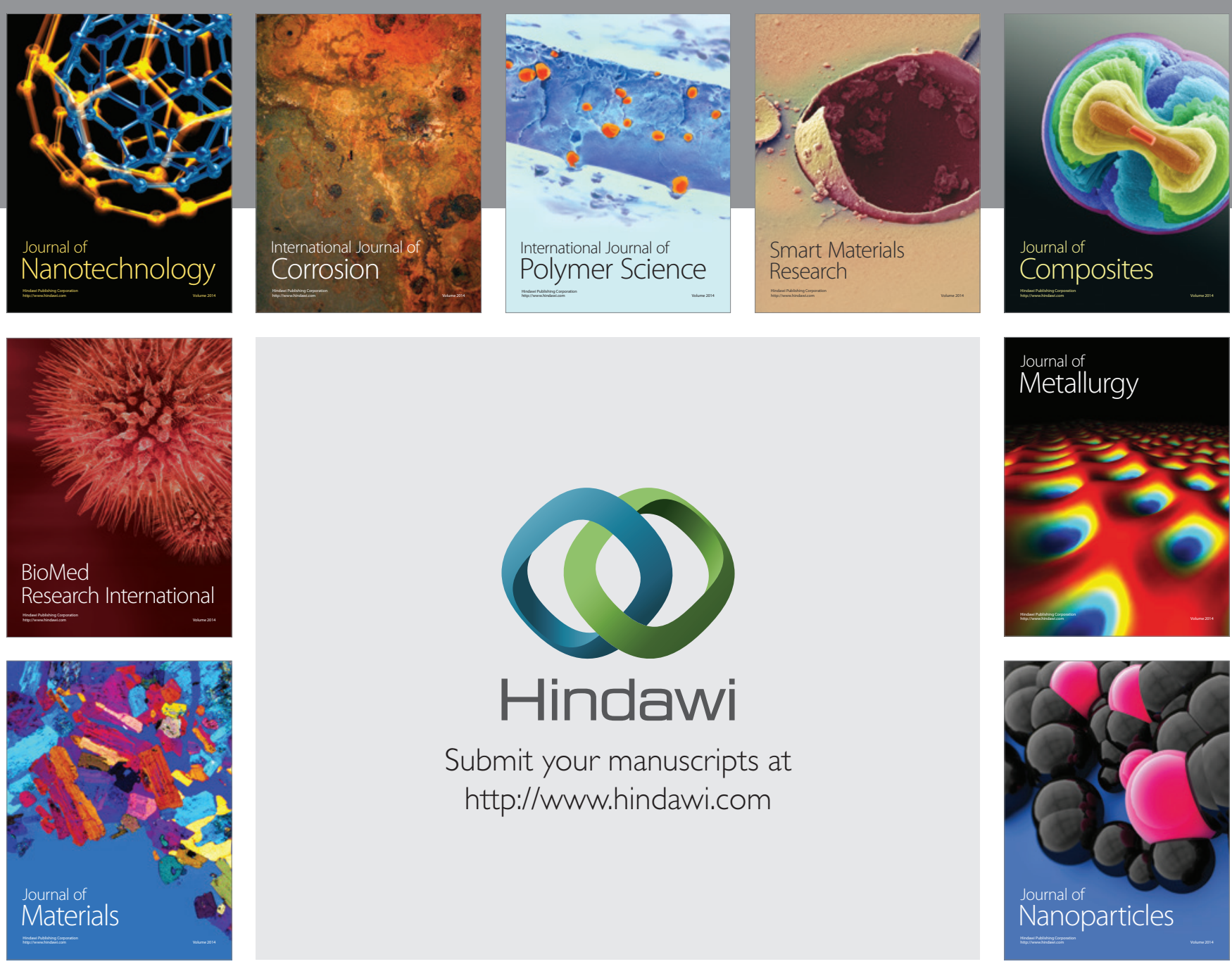

Submit your manuscripts at http://www.hindawi.com
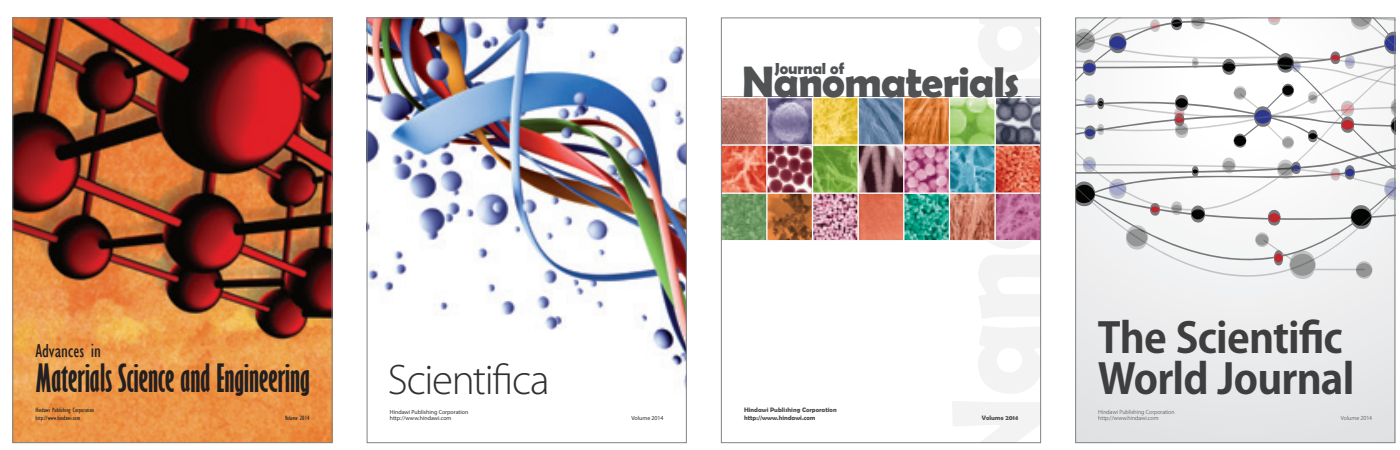

\section{The Scientific World Journal}
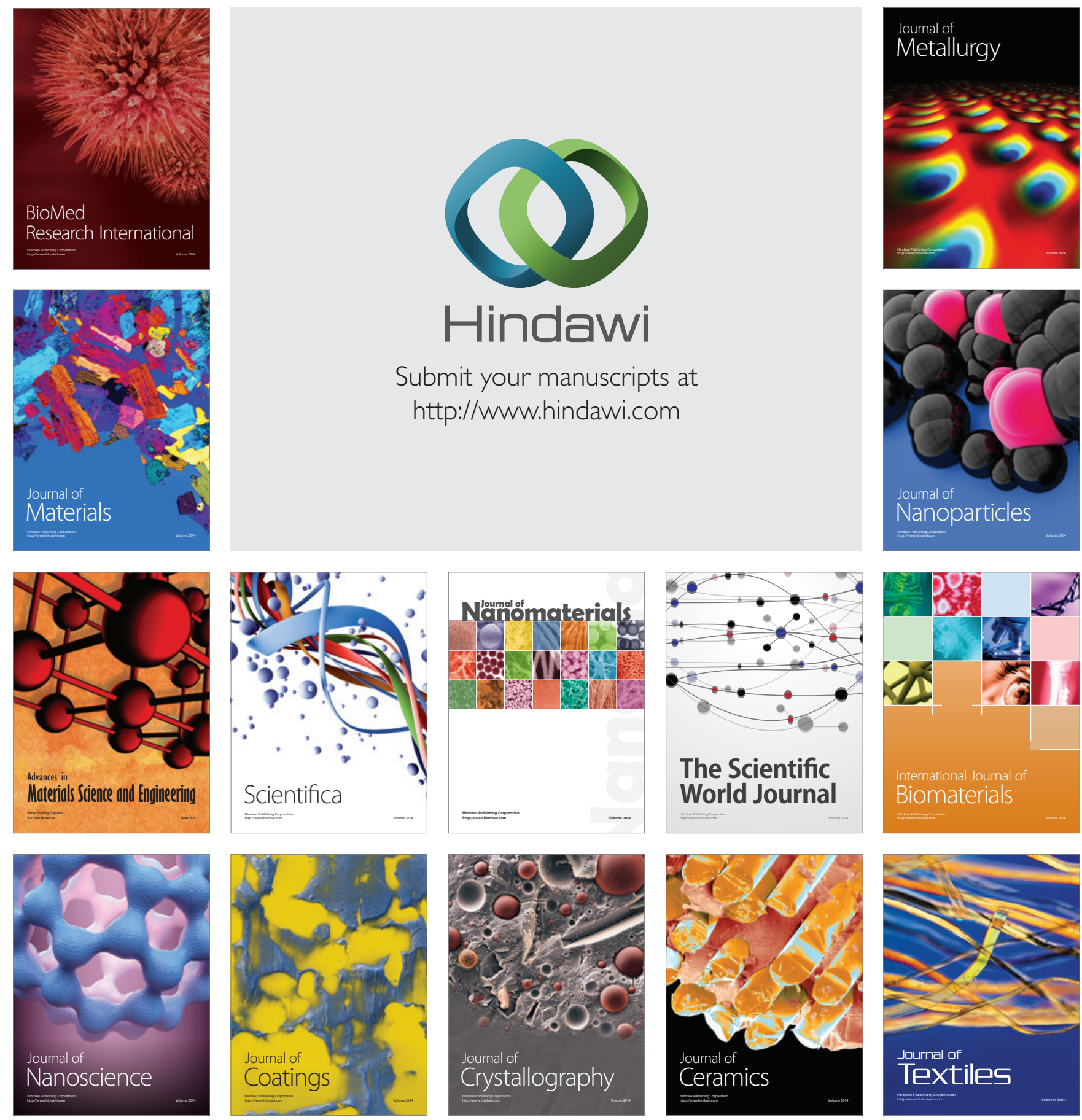